\title{
Often overlooked: formative evaluation in the development of ScienceComics
}

\author{
E. Weitkamp and H. Featherstone
}

\begin{abstract}
Formative evaluation should play a key role in the development of a science communication project or initiative. Such research is vital to understanding the needs and interests of the audience or participants; meeting these needs and interests helps ensure the project's success. However, there can be a temptation to plough ahead without undertaking adequate formative evaluation. Using ScienceComics (www.sciencecomics.uwe.ac.uk) as a case study, this article explores both the challenges and benefits of using formative evaluation to guide project development. It focuses on the actors involved in the formative stages and the impacts these actors had on the final outputs. This evidence is used to develop practical guidance on integrating formative evaluation right from the start.
\end{abstract}

\section{Context}

Many science communication initiatives start life in the minds of their creators. These initial ideas are translated into a funding application, where a target audience is specified and an outline of the project concept is provided. This initial project definition is informed by the past experiences of the creators, similar projects and evidence collected from other projects and experts, and may also be informed by specific pilot research that seeks to establish a need and audience for the initiative. However, once funding is obtained, how much further formative evaluation is needed to develop a robust project? And to what extent can and should the creators adapt and adjust the project in light of these initial findings? Taking a leaf from social marketing, this paper will explore how to use formative evaluation effectively to improve the overall success of the project.

Formative evaluation, incorporating both a review of previous projects and new evaluative work to inform the development of a new project or initiative, is recognised as critical to the success of social marketing programmes (see for example: $[1,2]$ ) where developing a message that the audience can relate to and which will have an impact on the target group is fundamental. Many of these programmes are seeking to change behaviours and understanding how your audience interacts with the information you are providing is crucial to achieving this behaviour change [3]. Formative evaluation can help the social marketer understand what motivates (or deters) an audience from adopting new behaviours; such information is crucial to gaining the participation or 'buy in' of the target group in the final initiative.

Science communication initiatives, while not necessarily seeking to change behaviours, also benefit from such an initial critical exploration of the project and how it meets the potential users' or audiences' needs. Indeed, NanoAventura, an exhibition designed to promote interest and curiosity in nanotechnologies, used just such an approach [4] and Joubert [5] advocates using formative evaluation at the start of a science communication project as a means of improving the project as it progresses. Most evaluation guides now highlight the need to build formative evaluation in from the start of a project (see for example: [6,7]). However, there is little documentation on the formative evaluation process for science communication and how this influences the finished project. 


\section{Defining Formative Evaluation}

Formative evaluation can be defined as the preparatory research that takes place in the early stages of project development. This initial research is typically undertaken in the start up phase of the project and as Patton [8] suggests, may be designed to:

"Improve implementation, solve unanticipated problems and make sure that participants are progressing toward desired outcomes." p. 69

As such, formative evaluation could helpfully be used to define aspects of the project as well as to improve the project as it develops. In the context of ScienceComics, formative evaluation was undertaken with the two key audiences for the project: teachers and young people aged 8-11. In working with these two distinct audiences, we sought to better understand the needs and interests of these users to allow us to develop an engaging resource for use in teaching science in primary schools.

\section{The ScienceComics project}

The initial idea behind ScienceComics was to support the teaching of the materials (chemistry) aspects of the National Curriculum for England. Previous research suggested that primary teachers lacked confidence in teaching science [9] and research indicated that humour was popular with both children and teachers and provided a memorable way of engaging with the science curriculum $[10,11,12]$. With this in mind, funding was obtained for a series of 10 comic adventures each with an associated teacher resource (demonstration, activity or investigation) which could be used by teachers for science lessons. The comics themselves might form part of the science lesson or indeed part of a literacy lesson and it was anticipated that these would be delivered either as a downloadable PowerPoint presentation or as PDF files formatted for viewing on screen. With increasing pressure on the primary curriculum putting a squeeze on the amount of time devoted to science [13], a cross curricular approach was anticipated.

As a result of the formative evaluation, the final resource (available free from www.sciencecomics.uwe.ac.uk) differs significantly from the initial project concept, which focused on delivering two elements: comic adventures and associated teacher support materials. Three key elements that changed as a result of the formative evaluation were: inclusion of interactivity within the comics, focus on scientific enquiry aspects of the curriculum (instead of simply focusing on the materials units) and provision of interactive games as part of an online package. The new resource is designed for use on an interactive whiteboard and consists of:

- ten comic adventures about Selenia, an alien with the ability to change the properties of materials

- ten linked scientific investigations

- $\quad$ quizzes that test pupils' knowledge of the science covered in the adventures

- $\quad$ games - word searches, sudoku and spot the difference puzzles

\section{Formative Evaluation Methods}

Early stages of the project focused on two audiences for ScienceComics: teachers and pupils. In order for ScienceComics to be used in classes, a resource was required that met the needs of UK primary school teachers, particularly those teaching in Key Stage 2 (Year 3-6, children aged 7-11). As gatekeepers determining children's access to the comics in class, teachers need to see a value in the resource and use it in the classroom. The other key audience for ScienceComics is children; teachers are more likely to use a resource that appeals to children as long as it also meets their teaching needs. Furthermore, many aspects of the project can be used by children independently of the teacher, whether in class or at home. For these reasons, formative evaluation was undertaken with both groups to ensure that the final product would meet teachers' needs, appeal to both teachers and children and finally engage young people with science. 


\section{Working with teachers}

Using local networks and schools, the initial phase of formative evaluation with teachers explored their needs and interests in relation to teaching science, the possibilities of using a comic in the classroom, the types of support materials they like to use or would like to use and the physical constraints and opportunities of the classroom, particularly in relation to the format of delivery of the final product. Tools for data collection ranged from questionnaires, which were useful particularly in identifying the best means of delivering the resource and the types of support materials they would like to see, to interviews following classroom visits. Information gained from questionnaires $(n=42)$ was used to develop prototype materials and activities which were then explored in more detail using interviews $(n=9)$ and in one focus group $(n=9)$. Teachers were involved in interviews on more than one occasion, so that their views on developments to the project could be obtained.

\section{Involving children}

Children had been closely involved in developing the pilot project, where they were involved in the selection of the illustrator for the project by reviewing artwork from three illustrators and indicating which character they would most like to read about and which they thought would have the best adventures [10]. This initial work highlighted the importance of involving children in the project development as much as possible, as they held strong views about the illustrations. It was also clear that choosing an illustration style that did not appeal to them would have greatly diminished their interest in reading the comics. Children's input was largely gained using whole class activities in the following areas:

- Creating and devising character profiles for two friends - what gender would the friends be (Selenia is female)?, What would her friends like to do? What hobbies would they have? What would their names be?

- Online activities - children were asked what kind of activities they would like to do online (e.g. games, quizzes) and paper prototypes were tested in the classroom

- Storyline development - using the pilot comic to set the scene, children were asked what other types of adventures they would like to read about

- Investigations - classes participated in trial investigations which provided insight into the abilities and interests of children in this age group

Formative evaluation with teachers and children was undertaken concurrently and iteratively. Thus, findings from the teacher questionnaire indicating that they would like a resource that could be used on an interactive whiteboard led to discussions with children about what types of activities they would like to do. This interconnectedness of the research was crucial in that it allowed the project to proceed along its original timescale, allowing elements to be fixed only at the point at which it was necessary for the project delivery schedule.

\section{Findings and application to the project}

\section{Balancing budgeting constraints}

Defining the resource was a crucial early aspect of the project. Results from the teacher questionnaire (figure 1) identified printed (book) and interactive whiteboard (IWB) formats as most popular. We did not have the budget available to produce a book, so focused our further research on the ways that IWBs are used in the classroom. Formative evaluation may suggest changes and options to a project team, but these may not all be feasible within the budget.

Teacher interviews indicated that the reason interactive whiteboards are popular in the classroom is because they are flexible, interactive and can be used to involve the whole class. Teachers gave examples of how they used interactive whiteboards which included: games, virtual experiments, animation, film clips and sound. Teachers also indicated that they accessed teaching materials through the internet. This opened up a number of opportunities for the project, such as the inclusion of interactivity within the comics as well as the potential to include interactive games on a website. 
Teachers' preferred formats for the comic adventures

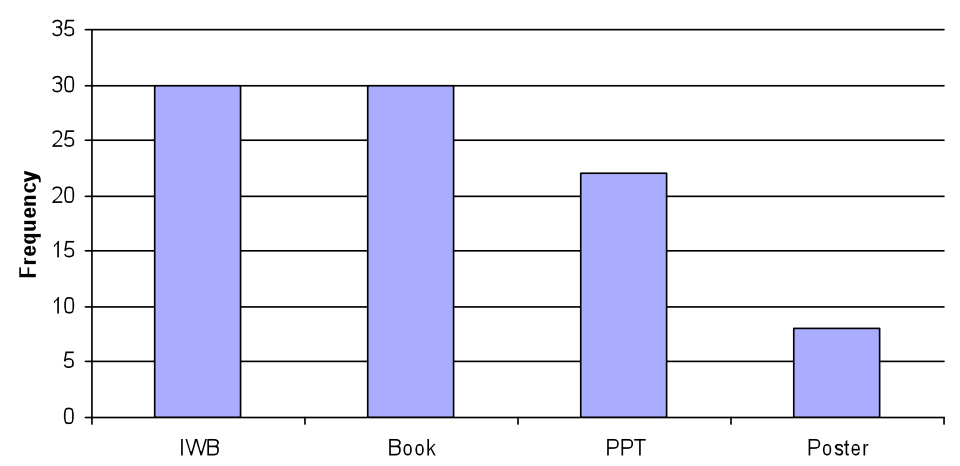

Figure 1. Format for delivery of Comic Adventures.

During formative evaluation, many options can be opened up to a project team, some of which will be more feasible than others. The challenge for the team is to keep focused, balancing challenges of implementation against benefits to the project. For example, several options were tested to make the comics interactive. In response to teacher feedback, sound effects were initially considered. However, the practicalities of getting these to work reliably in the classroom combined with the need for an unobtrusive way of indicating their presence to teachers, meant that this idea was discarded. However, teachers liked the idea of having questions in the comics which they could pose to their class (e.g. Why doesn't it [a gas dragon] hold its shape?, Episode 1 Selenia Starting Secondary School, page 5). Questions with pop-up answers could easily be marked through a change in background on the speech bubble. This gives teachers control: they can pose the question, get children to discuss it and if they wish click for the pop up answer. In some cases, the questions are also answered later in the comic through the action of the characters. Teachers also thought that having questions with pop-up answers would be a useful way of reinforcing scientific principles if children were using the comics independently.

Before fully committing to a course of action, a further round of formative evaluation may be needed. In the case of ScienceComics, a prototype comic was used to explore further whether the questions would be appreciated by both teachers and students. The students liked the questions because it gave them a chance to interact with the comic ("comics don't normally ask you anything, it gives you a chance to say things"). The teachers liked it because it gave them a chance to identify misconceptions (e.g. sand dissolves in water). The discussion allowed students to learn from each other (one student gave an accurate description of how sand forms and why it does not dissolve). The prototype comic included four questions and it became clear that this was too much (it became disruptive and repetitive). As a result the final resource has an average of two questions per comic.

New ideas developed during the formative evaluation can add value to a project if they can be incorporated within the existing budget and time constraints of the project. For example, creation of games was not envisaged in the original project plan. This limited the complexity of the games which could be produced, as only a small budget could be made available for games development. However, amongst the resources teachers suggested they would like were ideas for activities which are normally paper-based, like wordsearches (which promote the development of scientific vocabulary). Children were also asked about the types of activities they enjoyed doing in class and indicated word searches, crosswords and quizzes were popular. There was less familiarity with Sudoku type games, but children indicated an openness to try new games. These types of activities were also found on education websites identified by teachers. Children were clear though, that the games should be separate from the comics, not integrated (through for example clickable links) into the comics. This meant that they 'could just play the games without having to read through the comic every time you wanted to play them'. 


\section{Timing of formative evaluation}

Tempting as it may be to undertake formative evaluation as a separate stage of the project, with project development happening 'after the formative work is finished', this is not always possible. Careful consideration should be given to the timing of formative research, ensuring that information that is critical in the early phases of the project is available when needed. It became clear during the formative research for ScienceComics that more time should have been allocated early on to firming up how the whole package would work together. However, as the comic adventures take considerable time to illustrate, work needed to begin fairly early or the project would not have been delivered on time. Some issues had been anticipated and built into the formative evaluation, such as early identification of characters and storylines. However, the impact of moving to an IWB format meant some changes in style were difficult to incorporate and some opportunities missed (e.g. the placement of questions on the page was not as carefully thought through in the first few episodes).

In addition, during the later stages of the formative research when we focused more closely on the teaching support materials, it became clear that stronger links with the storylines would make the resources more appealing. As a result, we were not able to incorporate the suggestion of having a stopping point part way through an episode that would link directly to a classroom investigation. Instead, our investigations link more generally to difficulties encountered by the characters. For example, in Episode 5: Selenia in the Historical Adventure, Daiki is terrified of flying in a hot air balloon. The corresponding investigation challenges children to design a parachute to make him feel safer. In subsequent evaluation, teachers indicated that they liked the link between the comic and investigations.

'They are exactly what we need. It is good to get linked materials and they were easy to access and use.' - T05

We used formative evaluation to understand many issues which are often addressed through market research. For example, to explore the content and format of the comics, in terms of appeal and understanding. One class, asked to read Episode 1: Selenia Starting Secondary School, thought the comic was exciting and had interesting things in it (e.g. diamond doors). They also liked the cliffhanger at the end, something which was incorporated into each episode to encourage children to read on. Other aspects of the research were designed to investigate issues about the format of the comic: most children were able to navigate the pages (i.e. determine which frame to read next) without trouble and liked the questions posed. Some children indicated that they would prefer a printed comic and a small minority indicated that they did not enjoy comics, usually because they preferred more serious books.

Formative evaluation uncovered some interesting issues. Boys, for example, seemed to enjoy the comics more than girls. Boys were often first to raise their hands when asked to read frames of the comic or answer the questions posed. When readings were done with children sitting on the floor in front of the IWB, it was notable that boys tended to gradually creep forward during the reading and were very attentive throughout. Teachers commented that it attracted some children who would not normally read in class or respond to science. For example, one teacher commented 'pupils who usually don't answer science questions did answer on this occasion' and another that 'the boy who was keen to read aloud loves art and drawing but does not usually like science'.

\section{Dealing with strong opinions}

During the formative research, we encountered participants with great ideas and also strong views. In some cases, input from participants provided us with new insights and ideas we hadn't considered within the project team. For example, teachers were clearly of the view that they needed resources that were hands-on and would specifically support the Scientific Enquiry aspect of the National Curriculum [14]. These investigations would need to be practical (for large classes), cheap and interactive. The Scientific Enquiry element of the curriculum focuses on developing the investigation skills needed for scientific research, such as observation, planning, fair testing, data collection and presentation and drawing conclusions. However, questions remained about how prescriptive these investigations should be. A few of our informants were strongly of the view that the best teaching happens when teachers are inspired to develop their own materials. These informants felt that the investigations should provide a jumping off 
point and ideas for possible investigations. However, discussions with other teachers, such as science coordinators, indicated that many teachers were not sufficiently comfortable with science to take the risk of developing their own investigations. While most teachers did not advocate providing a 'recipe book' approach, there was a feeling that teachers did need enough information to test out an investigation. Within the project team, we found it quite difficult to strike a balance between these positions. In the end, we opted to provide guidance and basic instructions to teachers and to suggest extension ideas which the teachers could work up themselves.

The investigations proved to be one of the more challenging elements of the project: they needed to fit within the constraints of the school timetable, work with the storylines we were developing in the comic and be practical and engaging and the formative evaluation we undertook with classes and teachers was crucial to their development. Observation was used to understand which activities and approaches were most engaging for children and whether the tasks were too difficult or too easy. We also used classroom observations to judge how long each investigation took.

\section{Conclusions}

The extent to which a project can be adapted to accommodate the findings from formative evaluation depends on the flexibility of the project creators, budget and funder, amongst others. However, where this flexibility can be incorporated, formative evaluation can be used to shape and develop a more robust project. In the case of ScienceComics, we believe that the formative evaluation contributed to the development of a resource which is proving popular with teachers. By the end of the project, we had over 70,000 page visits and 7,000 unique visitors to the project website as well as many unsolicited positive comments from teachers.

The experience of using formative evaluation to shape the ScienceComics project suggests the following key requirements:

1. Project creators need to be flexible and open to accepting suggestions from participants in the formative evaluation

We were not able to incorporate all the ideas nor to accommodate all the suggestions. For example, several teachers suggested that they would like continuing professional development activities that would further support their teaching of scientific enquiry. However, there was not scope within the budget to deliver such training. As has been described here, where there was flexibility in the project we made changes to reflect the input from participants.

2. Build formative evaluation and flexibility into the project proposal

Building formative evaluation in at the start will ensure that you have the finances and time available to carry out the necessary research. While you may not be able to build in the budgetary contingencies that will allow large changes in the project direction, it is often possible to include some flexibility.

3. Ensure you involve all your key audiences

It would be easy to develop a resource that just appealed to one of our audiences (teachers or children or even the scientists involved or project funder). However, both teachers and children were key to the successful development of a resource for schools.

4. Consider how 'finished' the materials you test need to be

In many cases, participants in formative evaluation can provide useful input at very early stages of the project development when materials are in draft form. In the case of our comic stories, we found that children were able to provide feedback on the stories when they were simple sketches with text dropped on the page. Children would comment that there was too much text on a page, that they didn't understand an image or that the story didn't quite make sense. Similarly, both children and teachers were happy to comment on paper based versions of online resources, 
providing useful information (for example, word searches being too easy or hard or an investigation being too advanced or simple for a particular age group).

5. Undertake formative evaluation as early as possible

Practically, we could not undertake all the formative evaluation at the start of the project. This meant that some changes suggested at later stages could not easily and inexpensively be incorporated throughout all the comic adventures (in the case of this project, the timescale and costs were largely driven by the timetable for illustrating the comics themselves).

6. Know when enough research is enough

There is a temptation with formative evaluation to just keep going and collecting more evidence. At some point, you do have to get on and deliver the project. Although formative evaluation is an iterative process, we found that the information became saturated and that further research simply led to minor tweaks to materials. After this stage, analyse the information gathered and make a decision on the final shape of the project. ScienceComics generated interest amongst teachers and it would have been possible to carry on tweaking the investigations well past the end of the project.

7. Accept that you cannot please everyone

Throughout the project, but particularly during the development of the investigations, it was clear that there were strongly conflicting views. Science advisors, for example wanted more science and to avoid a 'recipe book' approach to the investigations. Children wanted fun activities. Teachers could be divided into two groups, those that wanted detailed instructions and those that wanted ideas that they could develop on their own. At some point, you as the creator have to make a decision on which group to please and recognise that you may not be able to please everyone.

8. Formative evaluation has positive impacts on participants

The children involved in this project REALLY liked being asked to participate in the creation of a resource for schools. Where schools were involved in multiple stages of the evaluation, children enjoyed the extended involvement and seeing how their ideas and opinions were incorporated. Teachers also indicated that they had enjoyed being involved in the project.

\section{Notes and references}

[1] L. Bellows, J. Anderson, P. Davies and C. Kennedy (2009), Integration of Social Marketing Elements in the Design of a Physical Activity Programme for Preschoolers, Social Marketing Quarterly 15(1): 2-21.

[2] S. Jones, S. Reis and K. Andrews (2009), Communication about Organ Donation Intentions: Formative evaluation for a Social Marketing Program Targeting Families, Social Marketing Quarterly 15(2): 63-73.

[3] S. Grier and C. Bryant (2005), Social marketing in public health, Annual Reviews of Public Health 26: 319-339.

[4] S. Murriello, D. Contier and M. Knobel (2006), Challenges of an exhibit on nanoscience and nanotechnology, Jcom 05(04):A01.

[5] M. Joubert, Evaluating Science Communication Projects, SciDevNet, nd http:/www.scidev.net/en/south-east-asia/practical-guides/evaluating-science-communicationprojects-1.html, accessed on 6 August 2009. 
[6] RCUK, Evaluation: Practical Guidelines, nd RCUK: Swindon, available online from: http://www.rcuk.ac.uk/cmsweb/downloads/rcuk/publications/evaluationguide.pdf, accessed on 6 August 2009.

[7] US Dept Health and Human Services, Making Health Communication Programs Work, nd, available online from: http://www.cancer.gov/pinkbook/allpages, accessed on 6 August 2009.

[8] M.Q. Patton, Utilisation Focused Evaluation: the New Century Text, $3^{\text {rd }}$ Edutuib, Sage, London (1997).

[9] C. Murphy, P. Neil and J. Beggs (2007), Primary Science Teachers Confidence Revisited: Ten Years on, Education Research 49(4): 415-430.

[10] E. Weitkamp and F. Burnet (2007), The Chemedian Brings Laughter to the Chemistry Classroom, International Journal of Science Education 29(15): 191-1929.

[11] P. Di Raddo (2006), Teaching Chemistry Lab Safety through Comics, Journal of Chemical Education 83(4): 571-573.

[12] L. Kruger and S.P. Watston (2001), Shoo - This Book Makes me to Think! Education, Entertainment and 'Life-skills' Comics in South Africa, Poetics Today 22(2): 475-523.

[13] B. Boyle and J. Bragg (2005), No Science Today - The Demise of Primary Science, The Curriculum Journal 16(4): 423-437.

[14] National Curriculum Online http://curriculum.qca.org.uk/key-stages-1-and2/subjects/science/keystage2/index.aspx?return=/key-stages-1-and-2/subjects/science/index.aspx, accessed on 20th July 2009.

\section{Author}

Dr Emma Weitkamp is a Senior Lecturer in Science Communication and a Science Writer. She is the author of Selenia's adventures as well as contributing to the other aspects of the project.

E-mail: emma.weitkamp@uwe.ac.uk.

Dr Helen Featherstone is a Research Fellow and the mastermind behind the games and investigations that form part of ScienceComics. E-mail: helen2.featherstone@uwe.ac.uk.

How TO CITE: E. Weitkamp and H. Featherstone, Often overlooked: formative evaluation in the development of ScienceComics, Jcom 08(04) (2009) A04. 